\title{
Method of Obtaining Malt Extracts
}

Viktoriia Khrystenko $^{1}$, Valentyn Chornyi ${ }^{2}$, Taras Mysiura ${ }^{3}$, Nataliia Popova ${ }^{4}$, Volodymyr Zavialov ${ }^{5}$

1. Department of Technology of Fats, Chemical Technologies of Food Supplements and Cosmetics, National University of Food Technologies, UKRAINE, Kyiv, Volodymyrska st., 68, E-mail: vika-akiv2804@ukr.net

2. Department of Processes and Apparatus of Food Production, National University of Food Technologies, UKRAINE, Kyiv, Volodymyrska st., 68, E-mail: val.chor@ukr.net

3. Department of Processes and Apparatus of Food Production, National University of Food Technologies,

UKRAINE, Kyiv, Volodymyrska st., 68, E-mail: taras_as@ nuft.edu.ua

4. Department of Foodstuff Expertise, National University of Food Technologies, UKRAINE, Kyiv, Volodymyrska st., 68, E-mail: nata_2506@nuft.edu.ua

5. Department of Processes and Apparatus of Food Production, National University of Food Technologies, UKRAINE, Kyiv, Volodymyrska st., 68, E-mail: zavialov@ nuft.edu.ua

Abstract - The work substantiates the expediency of using low-frequency mechanical vibrations for intensifying the extraction of target components from raw materials of grain origin. Optimal conditions and operating modes of the vibroextractor of periodic action for producing malt extracts are presented. The technology of obtaining dry malt extracts using the vibration extractor is given. Keywords - extraction, liquid-solid system, vibroextractor, malt, grain.

\section{Introduction}

Today, the market of dietary supplements is developing more rapidly, so obtaining an extract of malt plant materials with a high content of biologically active substances, and then entering it into foods, dietary supplements and cosmetics is relevant.

Among products of increased biological value, malt of cereal crops - wheat, oats, barley and corn - occupies a special place. Sprouted grains (malt) contain the entire set of components necessary for a balanced diet - proteins, easily digestible carbohydrates, dietary fibers, minerals, vitamins. In addition, malt cereals contain coloring and polyphenolic compounds, as well as plant enzymes and hormones.

Extracts are widely used as fillers and fortifiers of products; therefore, it is advisable to extract target components from natural raw materials by extraction.

\section{Materials and Methods}

At present, the practical provision of conditions for the optimal conduct of the extraction process with traditional methods is complicated, on the one hand, by the named and other properties of the raw materials, on the other hand, by the process-technological and constructive possibilities of the existing extraction equipment. Therefore, one of the relatively effective ways to intensify the process of extraction is the use of intensive energy-saving hydrodynamic modes, which are provided by low-frequency mechanical vibrations of the system.

Traditional technologies of periodic solid-phase extraction accompanies a number of disadvantages, among which are compaction of the mass of the raw material being processed and its simultaneous movement with rotating mixing devices and the like. This leads to a decrease in the relative velocity of the phases and the screening of the particles among themselves and, consequently, to a decrease in their activity in the process of mass transfer. Reducing or even complete elimination of the screening effect can be accomplished in the extractor, the principle of which involves continuous surface renewal phase contact method overlay medium interacting field low-frequency mechanical vibrations. For this purpose, the vibroextractor used a new 
principle of creating powerful vibro-turbulant flows in the working volume using flexible perforated vibration mixing devices.

The design feature of this vibratory extractor is possible to create turbulent pulsating alternating flows directed both toward the periphery of the apparatus and to the central part thereof. The hydrodynamic properties of these streams determine their action as a turbulizing factor at the micro level and a macroscale factor that eliminates stagnant zones.

The extract was extracted from several types of malt grain mass, namely: wheat malt, barley malt and corn malt.

The purpose of grain germination is the synthesis and activation of enzymes, under the influence of which the complete dissolution of all reserve substances is achieved in the extraction process. The significant content of enzymes in germinated grains of cereals contributes to the splitting of carbohydrates into simple sugars, and proteins into amino acids, that is, high-molecular and low-molecular water-soluble substances. Particularly interested in free amino acids that are directly assimilated by the human body.

Even under the action of enzymes, during germination, part of the complex substances of the grain is converted into maltose, glucose, maltodextrin and dextrins, peptones, peptides, amino acids and others.

\section{Results}

The hydromodule was selected for each raw material individually, and the normal composition of the malt grinding was characterized by the following ratios of fractions $\%$ : shells 15-18, large grits $18-22$, fine grits $30-35$, flour $25-35$. The ratio of fine grits and flour to large grits should be 3: 1 , but not less than 2.5: 1 . This is controlled by the excessive loss of the extract through the large grits.

The process is carried out, preventing contact with air. The optimal duration of the process is 255 minutes - for wheat malt (yield 3\%); 210 minutes - for barley malt (yield 2\%); 205 minutes - for corn malt (yield 2\%). Longer time keeping the malt in water is not advisable, since it does not lead to a significant increase in the solids content of the extract.

The production method of malt extract includes the cleaning of raw materials, grinding, extraction using low-frequency mechanical vibrations at a frequency of $5 \mathrm{~Hz}$, amplitude of 10 $\mathrm{mm}$, for the optimum duration of the process for a certain raw material and the temperature of the extractant $60-70^{\circ} \mathrm{C}$ and concentration. According to the technology, the vibrator-driven extractor has a vibration-turbulent mixing system. With a collection of filtered extract, the heater of the filtered extract is additionally connected in series, an evaporative unit, a collection of extract-liquid concentrate, a heater and dryer with a cyclone of an extract-liquid concentrate, powder extract concentrate conveyor, automatic machine for packing of extract concentrate powder, conveyor of packaged extract concentrate powder, and the collector-mixer of the meal of the extracted raw material and the sludge from the filtering centrifuge are additionally sequentially connected with the extractor and the filtering centrifuge, sludge press, sludge conveyor, sludge dryer with cyclone, dried sludge conveyor, automatic packaging machine for dried sludge and conveyor of packed sludge. 


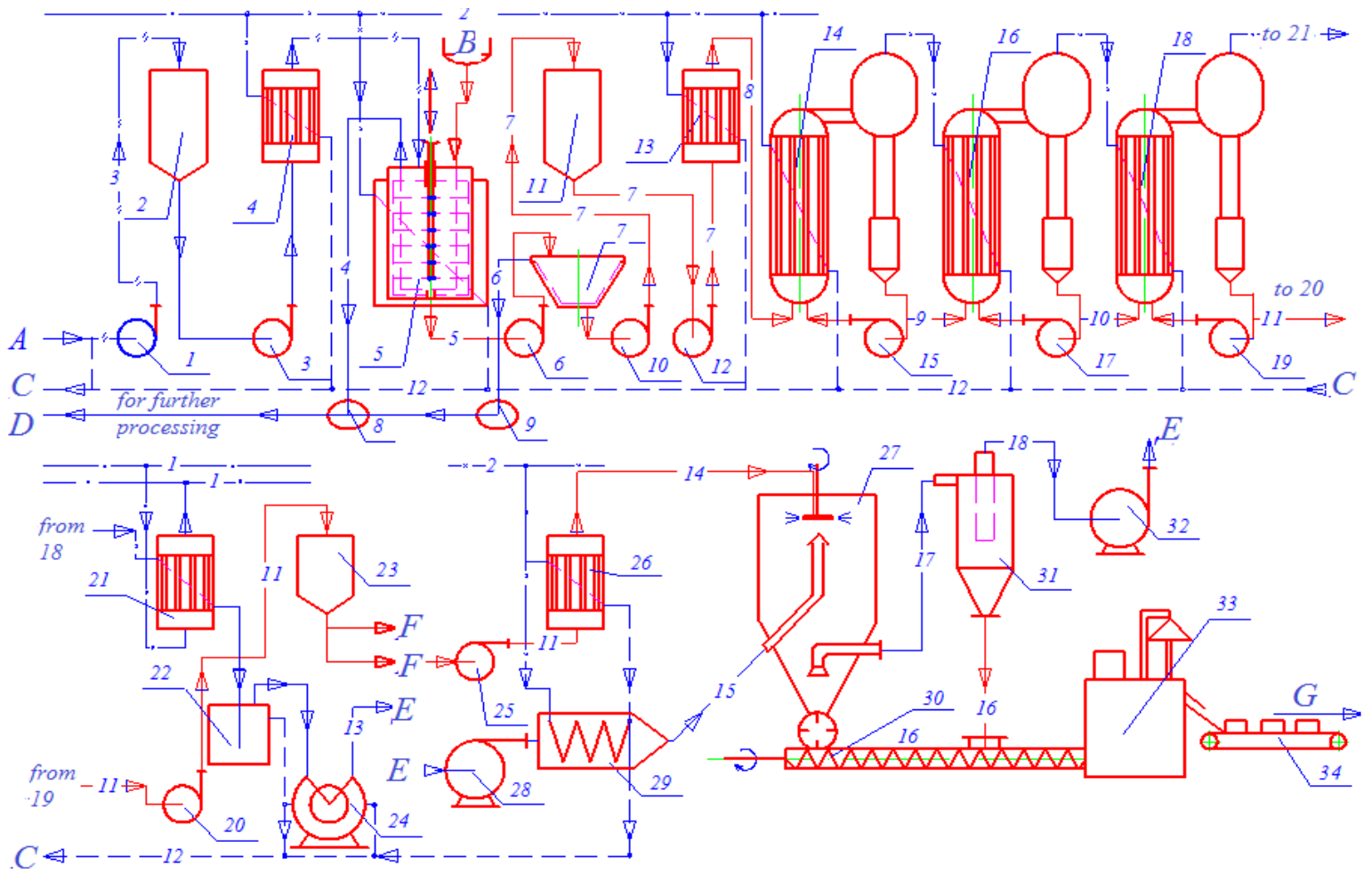

Fig.1. Hardware-technological scheme of production of extracts from plant raw materials by vibroextraction

2 - collector, 4 - heater of technological water - extractant, 5 - vibroextractor, 7 - centrifugal filter, 8 - collector of meal of extracted raw materials, 9 - collector of sludge from centrifuge, 11 - collector, 13 - filtered extract heater, 14, 16, 18 - three-body evaporator, 21 - secondary steam condenser, 22 - evacuated condensate collector, 23 - collector, 26 - concentrate heater, 1, 3, 6, $10,12,15,17,19,20,25$ - centrifugal pumps, 24 - vacuum pump, 27 - spray dryer, 28, 32 - fans, 30, 34 - conveyors, 29 - heater, 31 - cyclone of spray dryer 33 - packing machine.

\section{Conclusion}

The use of such an extractor will provide a high intensity of the extraction process, shorten its duration and ensure the maximum yield of biologically active substances from the plant material by reducing the stagnant fluid layer and creating convective flows that facilitate the transfer of substances to the extractant.

Thus, the modes of extraction of wheat, barley and corn malt were investigated, which allows to obtain high-value extracts from these types of raw materials for the cosmetic, food and pharmaceutical industries. Also developed a technology for producing malt extracts using vibroextraction equipment.

\section{References}

[1] B. E. Briggs, Linear Malts and malting. - London, UK: Springer Science \& Business Media, 1998.

[2] T. Mysyura, V. Zavialov, N. Popova, A. Rybachok, V. Chornyi, "Extractor," UA Patent 123314, 26 Feb., 2018.

[3] V. Zavialov, T. Mysyura, N. Popova, Y. Zaporozhets, V. Dekanskiy “ Investigation of hydrodynamics during continuous vibroextraction in a liquid-solid body system," Advances in Design, Simulation and Manufacturing. DSMIE 2018. Lecture Notes in Mechanical Engineering. Springer, Cham, pp. 524-535, 2019. 